\title{
Aerodynamic Drag Reduction for a Generic Truck Using Geometrically Optimized Rear Cabin Bumps
}

\author{
Abdellah Ait Moussa, Justin Fischer, and Rohan Yadav \\ Department of Engineering \& Physics, University of Central Oklahoma, 100 North University Drive, Edmond, OK 73034, USA \\ Correspondence should be addressed to Abdellah Ait moussa; aaitmoussa@uco.edu
}

Received 8 September 2015; Revised 6 November 2015; Accepted 15 November 2015

Academic Editor: Éric Serre

Copyright ( 2015 Abdellah Ait moussa et al. This is an open access article distributed under the Creative Commons Attribution License, which permits unrestricted use, distribution, and reproduction in any medium, provided the original work is properly cited.

The continuous surge in gas prices has raised major concerns about vehicle fuel efficiency, and drag reduction devices offer a promising strategy. In this paper, we investigate the mechanisms by which geometrically optimized bumps, placed on the rear end of the cabin roof of a generic truck, reduce aerodynamic drag. The incorporation of these devices requires proper choices of the size, location, and overall geometry. In the following analysis we identify these factors using a novel methodology. The numerical technique combines automatic modeling of the add-ons, computational fluid dynamics and optimization using orthogonal arrays, and probabilistic restarts. Numerical results showed reduction in aerodynamic drag between $6 \%$ and $10 \%$.

\section{Introduction}

With budgets tight, gas prices on the rise, and fuel economy on everyone's mind, it is now more imperative than ever to invest in new energy-saving technologies among all products and services, including more energy efficient vehicles. In the automotive industry, trucks are known for their relatively higher drag coefficients [1-8] which suggest that there is room for improvement.

Previous studies performed on academic geometries showed that proper modification of the flow topology around a vehicle can improve its aerodynamic performance by reducing drag [4-8]. Flow control devices, such as cabin flaps [6], continuous suction, and/or blowing solutions [9-14], were proven to offer promising results. A carefully designed cabin flap, for instance, can increase the cabin surface pressure coefficient and displace or even eliminate the attachment of the bed flow on the tailgate, eventually reducing the size of the reverse flow in the wake [6]. Bump-shape vortex generators [15], commonly used on aircraft to delay flow separation, are another promising strategy that does not require major changes in vehicular geometry. These devices were analyzed for applications on the roof end of sedan vehicles and have proven to delay flow separation above the rear window which led to reduction in aerodynamic drag [16].
In this paper, we investigate the mechanisms by which geometrically optimized bumps, placed on the rear end of the cabin roof of a generic truck, reduce aerodynamic drag. These devices are expected to deflect the flow behind the cabin back slightly downward, eventually displacing the flow attachment over the tailgate which may lead to improvement in the pressure coefficient over the cabin back and the outside surface of the tailgate similar to that of the cabin flap [6]. Unfortunately, these devices may generate additional drag which could otherwise raise the overall drag coefficient of the vehicle. Accordingly, careful design, location, and multiplicity must be adequately selected for maximum improvement in drag reduction.

To numerically identify the aforementioned design parameters in a self-regulated manner, we automated the design and simulation processes and allowed a search algorithm to create a final design that meets the particular requirements and minimize the drag coefficient. The methodology, as indicated, will be emphasized in more detail in the next few sections. Furthermore, design parameters, whether physical or geometrical, may vary over several constrained continuums and the selected search method should be able to simultaneously navigate over the design space of each variable and promptly produce the best optimal configuration. This conception is further investigated and a novel search 


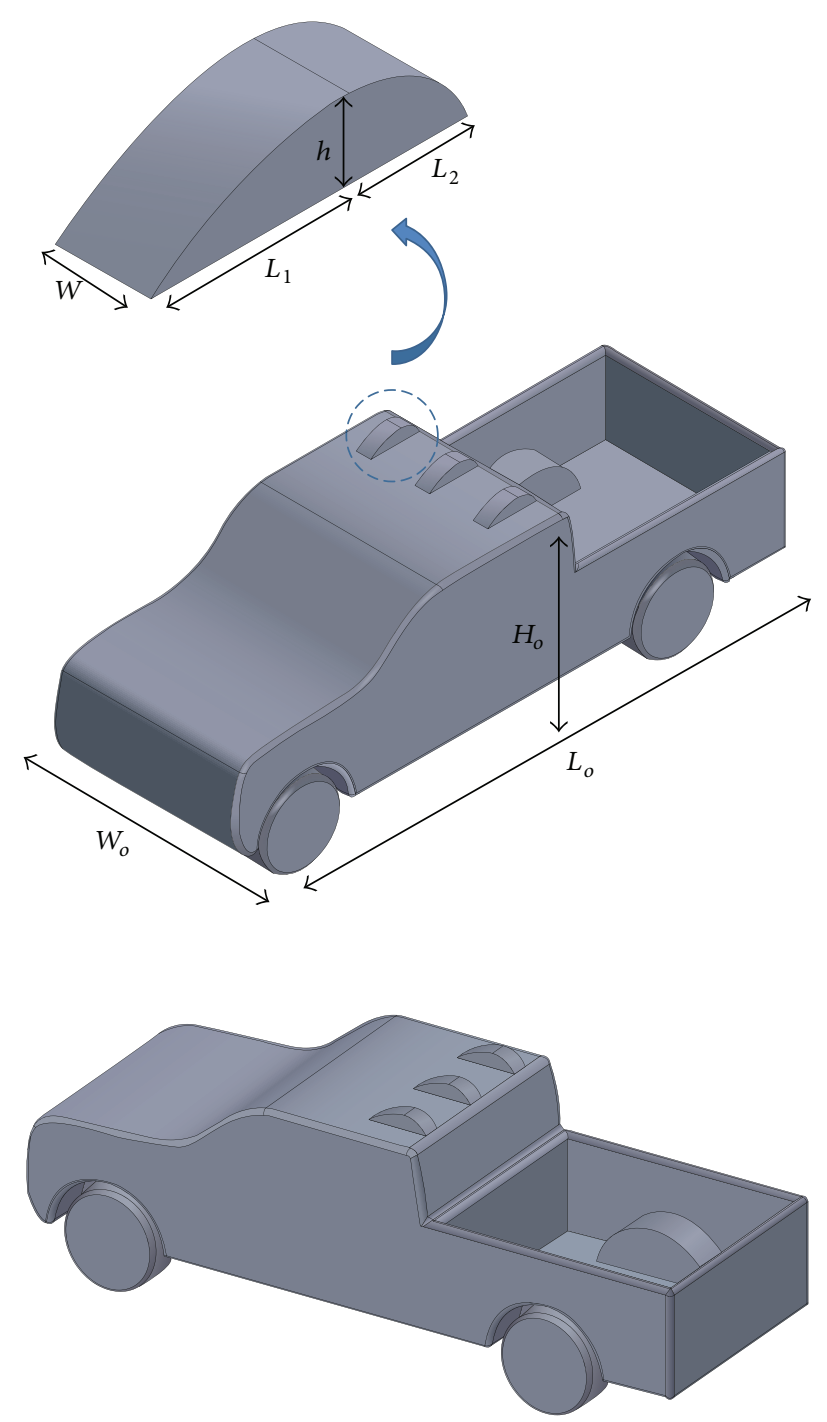

FIgURE 1: Physical model with 3 bumps.

method that uses orthogonal arrays, a probability density, and multiple restarts is introduced.

This paper is divided into three major parts. In the first, we introduce the technique of optimization and components used in the process of computing and minimizing aerodynamic drag. Next, we benchmark and apply this technique to a generic model of a commercial truck customized with rear cabin bumps. We conclude with a summary and a discussion of future work.

\section{The Computational Technique}

In this section, we shall describe the general structure of the computational technique that we apply to a specific model of a generic truck.

2.1. The Physical Model. A pictorial representation of the truck model used in the analysis is shown in Figure 1.
The physical model is $1 / 10$ th scale generic truck without side mirrors. The overall length $\left(L_{o}\right)$, width $\left(W_{o}\right)$, and height $\left(H_{o}\right)$ were $L_{o}=450.8 \mathrm{~mm}, W_{o}=150 \mathrm{~mm}$, and $H_{o}=148.4 \mathrm{~mm}$, respectively. A number of bumps ( $n=3$ in the figure) are added on the surface of the cabin. The dimensions of one of the bumps are displayed on the inset of the same figure. The sketch is a combination of two adjacent arcs extruded along the normal direction over a known width. The projected lengths $\left(L_{1}, L_{2}\right)$, the width $(W)$, and height $(h)$ along with the number of bumps $(n)$ are the design variables that will later be identified using the technique introduced in the following section. Note that the top and bottom half of the back surface of the cabin are on two separate planes; they are joined however in the model with a set of fillets. Furthermore, the bumps are set up to be equally spaced and symmetrical about the symmetry plane of the truck. More specifically, for an " $n$ " number of bumps, the spacing was equal to ratio of the width of the truck and the number of bumps (i.e., $\left.W_{o} / n\right)$. In addition, for even values of " $n$," the bumps are 
sparse symmetrically on both sides of the symmetry plane. For odd values of " $n$," the geometrical center of the central bump is right on the symmetry plane; the rest of the bumps are symmetrical with reference to the symmetry plane as depicted in Figure 1 for $n=3$.

2.2. The Optimization Technique. In the previous section, we identified five design parameters $\left(n, W, L_{1}, L_{2}\right.$, and $\left.h\right)$ that characterize the geometry and multiplicity of the bumps. In this section, we introduce the optimization technique that will be utilized in Section 3 to identify the values of these factors for maximum reduction in the overall aerodynamic drag.

Orthogonal array optimization, or Taguchi method [17, 18], is a statistical technique used to study the simultaneous effect of multiple variables on the performance of a process. It was developed by Genichi Taguchi from Japan during late 1940. Taguchi showed that design of experiments (DOE) could be used to improve the quality of manufactured products. He suggested that fractional factorial design of experiments provide a mean to cost-effectively investigate complex problems. Taguchi devised a number of special orthogonal arrays (OA) that allowed statistical analysis of results with high degree of confidence. He suggested a way to analyze the experimental data and identify the best quality process to be used based on minimizing the deviation from the target value. Taguchi method was successfully used in several research areas [19-22] and in the current development, this method is utilized to identify the set of design parameters, $(n$, $\left.W, L_{1}, L_{2}, h\right)$, that minimize the overall aerodynamic drag.

Orthogonal array optimization requires, in addition to the design variables (factors), a list of levels for each factor. The number of factors and their levels determine the orthogonal array to be used. In the current analysis, three levels were identified for each factor and an $L_{18}$ orthogonal array was utilized. Since all factors, but the number of bumps $(n)$, can vary over constrained continuums, opting to identify the best optimum this way may not lead to the best solution. A global search however can be conducted by restarting the Taguchi algorithm over the domain of analysis of all design variables. The methodology schematics are shown in Figure 2. To be able to navigate over the domain of each factor, we begin by generating a number of initial vertices, where each vertex encompasses random values of each of the design variables $\left(x_{i}^{o}\right)$; we then use the variable variance probability density $[23,24]$ in the Appendix to identify a vertex that is far from all initial vertices. The next two levels of each factor in the Taguchi table $\left(x_{i}^{1}, x_{i}^{2}\right)$ were calculated according to (1), where $\left(\Delta x_{i}\right)$ refers to the size of the domain of analysis of variable $\left(x_{i}\right)$. We then proceed with Taguchi method and identify the most optimal combination of levels. To avoid selecting the same experiments when restarting the Taguchi algorithm, we add the optimal solution to the list of initial vertices and then restart the search by selecting the vertex with the largest distance from all initial vertices and known optimal solutions. There may be cases however, when the new optimum is identical to one of the stored optima, that the suggested optimum is not better than one of the current vertices because of factor interactions or that one or more of the vertex levels are not on the box constraint. In cases like these, we proceed as indicated in the diagram of Figure 2. The box projection procedure in (2) ensures that the levels are always selected over the domain of the analysis. Consider

$$
\begin{aligned}
x_{i}^{k} & =x_{i}^{o}+0.1 \times(-1)^{k} \Delta x_{i}, \quad k=1,2, \\
x_{i} & = \begin{cases}x_{i}^{\text {lower bound }}, & \text { if } x<x_{i}^{\text {lower bound }}, \\
x_{i}^{\text {upper bound }}, & \text { if } x>x_{i}^{\text {upper bound }},\end{cases}
\end{aligned}
$$

where $x_{i}$ is a level sampled during the optimization.

2.3. Program Structure. To achieve optimal values of drag coefficient (Cd) [1], we will be facing three parts of work: geometric modeling, finite element analysis (FEA), and mathematical programming. Different program files were developed for each part, and communication between the different entities is controlled by an interface. The methodology schematics are shown in Figure 3. The mathematical programing entity includes the random generation of the initial vertices, the self-regulated calculation of the probability density for each vertex, and the automatic construction of the $L_{18}$ orthogonal array used in the optimization. These instructions are implemented in an Excel Visual Basic Editor (VBE) and the results of the respective calculations are displayed in the main sheet of the accompanying workbook. To design the bump-shape add-ons, we used SolidWorks (solid modeling Computer-Aided-Design (CAD) software that runs on Microsoft Windows). This software also comes with a Visual Basic for Application (VBA) toolset that allows one to record, run, and edit the different SolidWorks Application Programming Interface (API) calls. This set of commands were then imbedded in the main Excel VBA Macro as a user defined function that is called every time design updates are generated. A parasolid model of the updated truck geometry is saved in an external file as a result of this operation.

For the finite element analysis (FEA), we used the ANSYS Workbench, where ANSYS is FEA software used to calculate the objective function and constraints. Communication with ANSYS is made possible via a Workbench journal file. Commands for uploading the parasolid model of the truck with add-ons, for adding an enclosure to model fluid flow around the vehicle, were incorporated in an external file using the Java Python language for the ANSYS Design Modeler. Commands for meshing, for adding inflation on the road and truck surfaces, and for applying body sizing and named selections were incorporated in an external file using the Java Python language for the ANSYS Mesher. Commands for initializing the Fluent computation and applying boundary conditions were incorporated in a Fluent journal file. Upon completion of the pre- and postprocessing stages, ANSYS provides results file which records the drag coefficient $(\mathrm{Cd})$ over the steps of the simulation; this information is stored in an external file and returned to the main Excel VBA Macro.

2.4. Finite Element Analysis Setup and Validation. We used the Fluent analysis system in ANSYS Workbench. The model, 


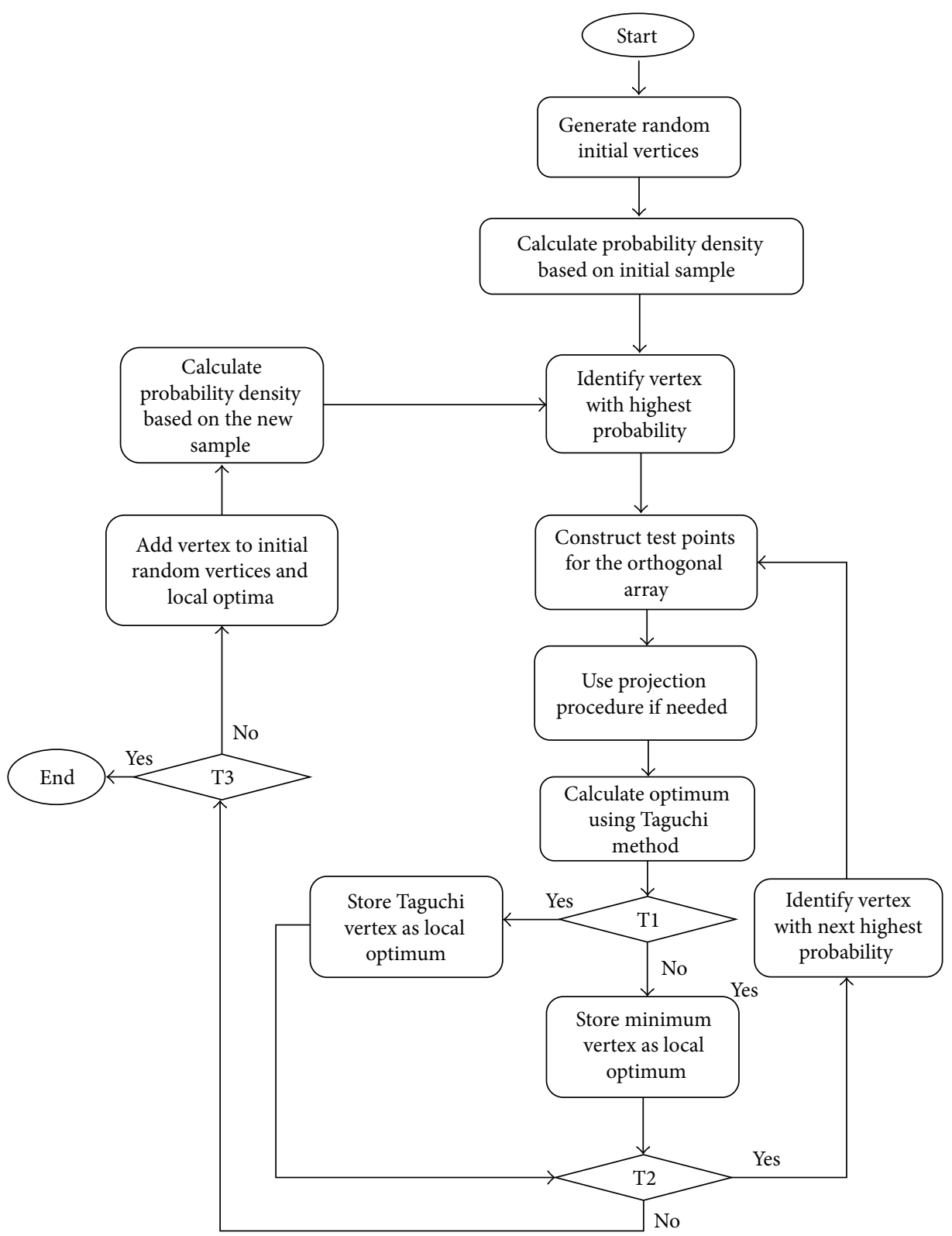

Figure 2: Global optimization. T1: Taguchi suggested optimum is best, T2: already known as an optimum, and T3: the maximum number of analyses is reached.

including the bumps, was imported to the Design Modeler and aligned with a control volume. A half model was used to allow quicker solution of the model with a more refined mesh. The control volume size was set according to Fluent's best practice guide for vehicle analysis [25]. The computational domain in Figure 4 extended around three times the vehicle length to the front and five times to the rear. A box was created around the vehicle and in the wake region to control the mesh size during the meshing process. The box extended about half a vehicle length in front, to the sides, and to the top and about a vehicle length in the wake. The model was then subtracted from the computational domain to limit the computational analysis to the rest of the control volume and vehicle boundaries.
In this investigation we adopted a physics based meshing in the ANSYS Mesher; the physics preference was set to CFD and solver to Fluent. An inflation layer was added over the surfaces of the vehicle and the road as shown in Figure 5; the prisms were grown with a first aspect ratio of 10 and a growth factor of 0.9 extruding 7 layers. This setting was proven to ensure a good transition in terms of cell-size deviation at the interface between the prismatic layers and the tetrahedral regions. Body sizing was used for mesh refinement around the vehicle and wake region. Triangular mesh elements were used on the surface to reduce the numerical diffusion and to align with the real flow near the model. The remainder of the computational domain was filled with tetrahedral volume cells that were adjacent to the prism layers. 


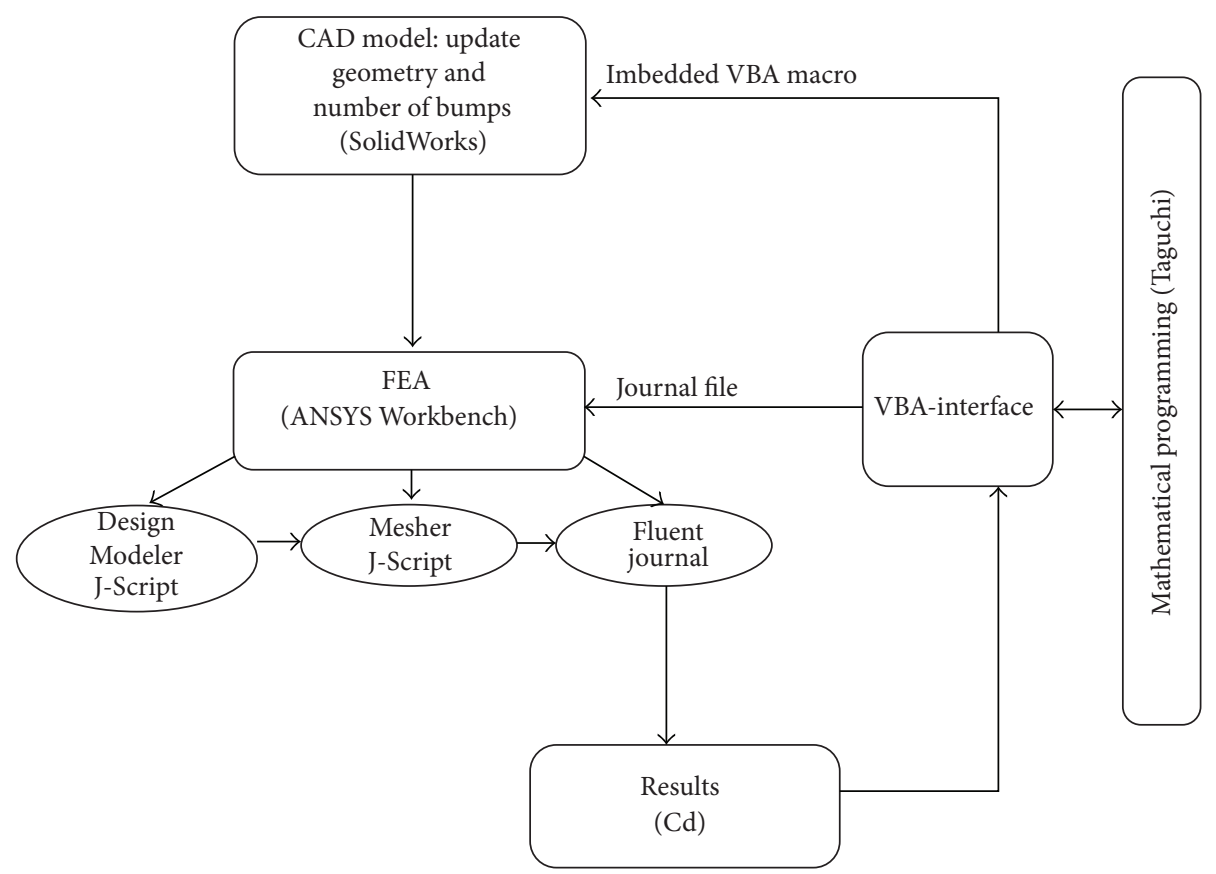

Figure 3: Program structure.

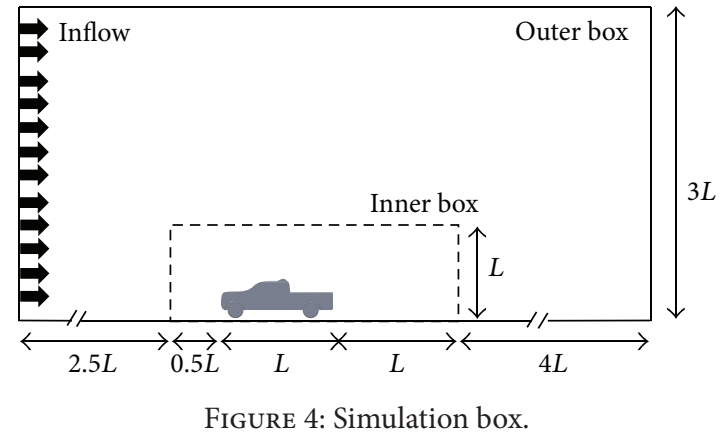

A velocity-inlet boundary condition was used to model the incoming flow. Fluent's best practice guide for vehicle analysis [25] recommends using a realizable $k$-epsilon model and nonequilibrium wall functions (NWFs). The physics parameters are summarized in Table 1 . Fluent convergence criterion of $10^{-4}$ for the continuity and momentum equations was used.

To validate the meshing and physics model used in this simulation, we utilized the experimental results of Al-Garni et al. [8] conducted on a truck model similar in size and geometry to the truck in Figure 1 (without bumps). From the simulation results, we plotted the change in the pressure coefficient $\left(C_{p}\right)$ over the symmetry plane over the top and bottom surfaces of the plain model in Figure 6. The results from the simulation compare well to the results given in the experimental study of Al-Garni et al. This validates the meshing and physics models being used for the simulation of bluff bodies in this work and provides strong evidence for the validity and accuracy of the results obtained from the simulations. The pressure coefficient in the plot is defined in
(3), where $(A)$ is the projected area of the vehicle, $(\rho)$ is the density of the ambient air, $v$ is the free stream velocity, $(p)$ is the pressure, and $\left(p_{\infty}\right)$ is the pressure in the free stream:

$$
C_{p}=\frac{p-p_{\infty}}{(1 / 2) \rho A v^{2}}
$$

2.5. Grid Independence Test. A grid independence test was performed on the geometry without add-ons, and the drag coefficient and convergence time were selected as the criteria. Six nodes were used in parallel computation to conduct the FVA simulation. Referring to the results in Table 2, there is a variation of less than $5 \%$ between medium and finest mesh, and as such, the results are assumed to be largely grid independent. Balance between calculation, time, and the accuracy order of the simulation has been made and the setting for the "Finel" grid is considered to be sufficiently reliable. 


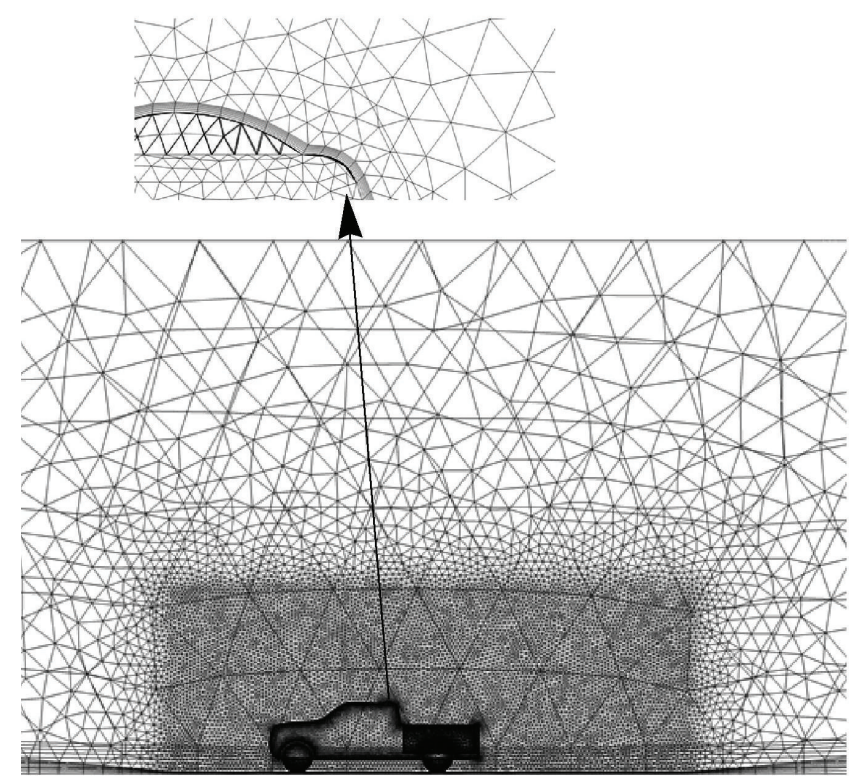

Figure 5: Boundary layer at a growth factor of 0.9, triangular mesh elements on the surface, and tetrahedral volume cells in the remainder of the computational domain. The inset displays the inflation layer on the surface of one of the bumps.

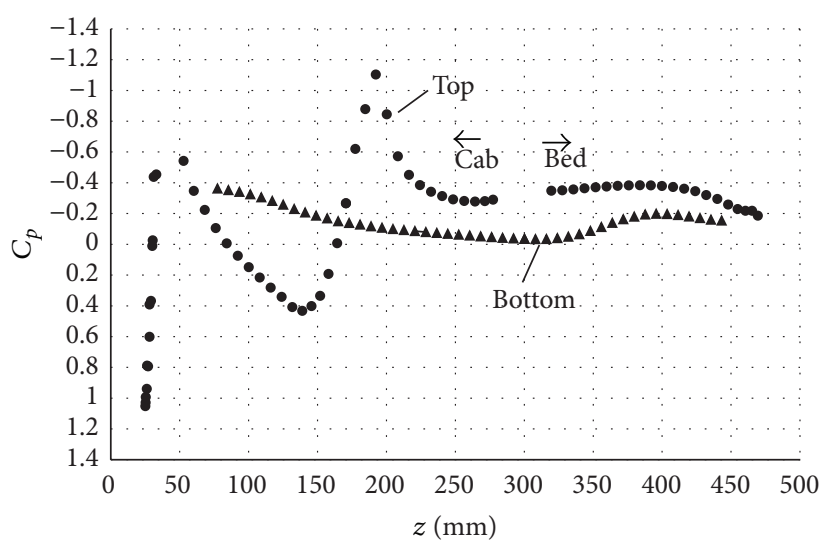

FIGURE 6: Pressure coefficient distribution on the symmetry plane of the truck model in Figure 1.

\section{Results and Discussion}

In this section we shall discuss the results of the application of the technique introduced in Section 2 to the test model in Figure 1. A discussion over the final results is also included.

3.1. Optimization Setup and Results. The methodology introduced in Section 2 was applied to the model truck configuration in Figure 1. The box constraints for the design parameters were set according to (4). The free stream velocity was set to $v_{\infty}=30 \mathrm{~m} / \mathrm{s}$, and the Reynolds number calculated based on the overall model length was $\operatorname{Re}=7.95 \times 10^{5}$. We started with 10 random initial vertices over the box constraints, and the optimum points were rounded off to $10^{-3}$. The maximum number of repetitive restarts was set to 30 . Consider

$$
\begin{aligned}
2 & \leq n \leq 7, \\
0.04 & \leq \frac{W}{H_{o}} \leq 0.094,
\end{aligned}
$$

$$
\begin{aligned}
& 0.07 \leq \frac{L_{1}}{H_{o}} \leq 0.40, \\
& 0.07 \leq \frac{L_{2}}{H_{o}} \leq 0.11, \\
& 0.03 \leq \frac{L_{2}}{H_{o}} \leq 0.07 .
\end{aligned}
$$

The values of the drag coefficients were calculated for each factor set in the orthogonal arrays, and the average values of the drag coefficient over the last twenty steps were reported. Although we had around 18 different configurations that a designer can choose from, not all contributed equally to the reduction of the drag coefficient. Table 3 summarizes 6 of the 18 configurations pertaining to different values of the number of bumps $(n)$; the last column displays the percent reduction in aerodynamic drag coefficient over to the plain 


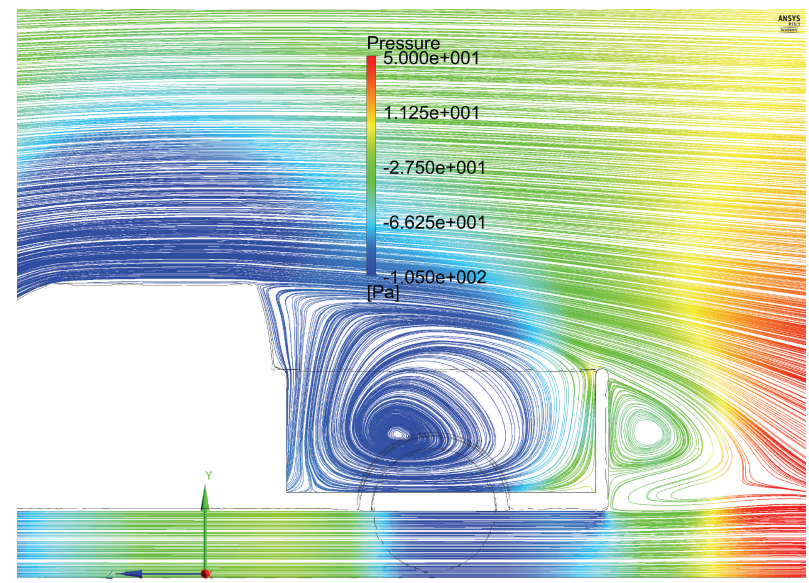

FIGURE 7: Streamlines colored by the pressure in the symmetry plane for the truck without add-ons.

TABLE 1: Physical parameters.

\begin{tabular}{ll}
\hline Physical properties & Parameters \\
\hline Flow characteristics & $\begin{array}{l}\text { Steady, stationary, incompressible, } \\
\text { turbulent, segregated flow with } \\
\text { energy solver, RANS }\end{array}$ \\
\hline Solver type & Pressure-based \\
\hline Turbulence model & $k$-epsilon, realizable \\
\hline Near-wall treatment & Nonequilibrium wall function \\
\hline Inlet flow velocity & $30 \mathrm{~m} / \mathrm{s}$ \\
\hline Outlet pressure & 1atm \\
\hline & $\begin{array}{l}\text { (i) Pressure-velocity coupling: } \\
\text { SIMPLE scheme } \\
\text { (ii) Spatial discretization: gradient } \\
\text { (least square cell based), pressure } \\
\text { (standard), momentum } \\
\text { (Second-Order Upwind), turbulent } \\
\text { kinetic energy (Second-Order } \\
\text { Upwind), turbulent dissipation rate } \\
\text { (Second-Order Upwind) }\end{array}$ \\
\hline
\end{tabular}

TABLE 2: Grid independence test.

\begin{tabular}{lcc}
\hline Total number of cells & Drag coefficient & Convergence time \\
\hline $\begin{array}{l}\text { Medium } \\
(1,322,679 \text { million })\end{array}$ & 0.442 & 110 minutes \\
\hline $\begin{array}{l}\text { Fine1 } \\
(3,012,125 \text { million})\end{array}$ & 0.449 & 215 minutes \\
\hline $\begin{array}{l}\text { Fine2 } \\
(6,022,233 \text { million})\end{array}$ & 0.451 & 420 minutes \\
\hline
\end{tabular}

configuration without add-ons. The numerical results show an improvement between 6 and $10 \%$ for different bump geometries and multiplicity configurations.

3.2. Discussion of Results. To understand the underlying causes of this improvement, we first displayed the streamlines inside the bed and behind the tailgate over the symmetry plane for the truck geometry without add-on in Figure 7 and
TABLE 3: Optimum design obtained by orthogonal arrays.

\begin{tabular}{cccccc}
\hline$n$ & $W / H_{o}$ & $L_{1} / H_{o}$ & $L_{2} / H_{o}$ & $h / H_{o}$ & \% reduction \\
\hline 4 & 0.040 & 0.267 & 0.082 & 0.031 & 8.67 \\
2 & 0.081 & 0.308 & 0.100 & 0.029 & 9.05 \\
7 & 0.071 & 0.073 & 0.094 & 0.031 & 6.47 \\
6 & 0.073 & 0.400 & 0.104 & 0.036 & 8.88 \\
5 & 0.093 & 0.183 & 0.093 & 0.027 & 9.03 \\
3 & 0.088 & 0.334 & 0.078 & 0.062 & 9.83 \\
\hline
\end{tabular}

the optimal configuration with two bumps in Figure 8. The colors of the lines represent the pressure distribution. The recirculating flow over the bed is fairly noticeable. This flow was detached from the rear end of the cabin roof and entered the inside of the bed in a clockwise direction. In the wake, the reverse flow appeared as the wake flow of a bluff body. We calculated the pressure coefficient $\left(C_{p}\right)$ over the symmetry plane, on the outside surface of the tailgate in Figure 9, and on the back surface of the cabin in Figure 10 for the configurations with an even number of bumps $(n=2,4,6)$. There is a noticeable improvement in the pressure coefficient on the outside surface of the tailgate for all configurations when compared to the plain geometry without add-on. We also noticed a similar improvement on the cabin back.

After careful analysis of the flow over the cabin and the bed, we realized that the bumps had deflected the flow downward and noticeably steeply next to the cabin back as illustrated in Figure 11, where we compare the velocity components over the planes labeled 1, 2, and 3 in Figure 12 for both the plain configuration (left column in Figure 11) and the configuration with two bumps (right column in Figure 11). The local coordinate frame shown on the same figure is at the same height as the cabin roof. The color bands on the top slide indicate that the velocity component over the plane is negative (pointing toward negative $Y$-direction) with magnitudes - as indicated by the size, color, and order of the layers - that are noticeably larger for the configuration with bumps than it is for the plain configuration (i.e., for equal $Y$-values, the magnitude of the velocity component is larger 


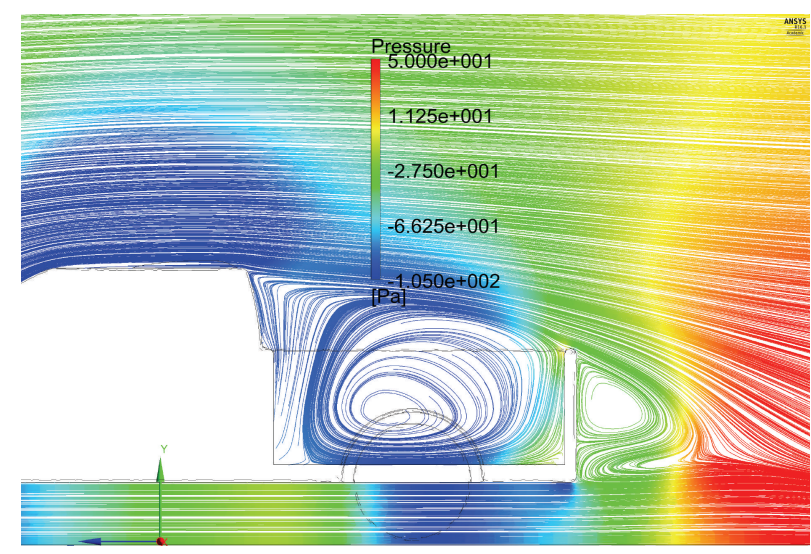

FIGURE 8: Streamlines colored by the pressure coefficient in the symmetry plane for the truck with two bumps.

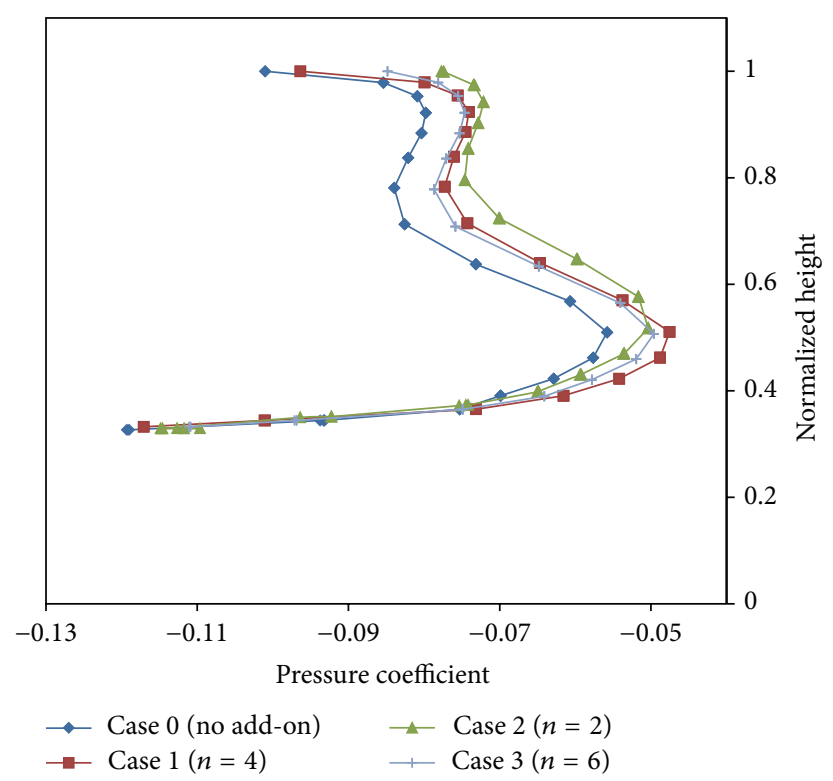

FIGURE 9: Pressure coefficient on the back surface of the tailgate. (Origin of the axes is displayed in Figures 7 and 8. )

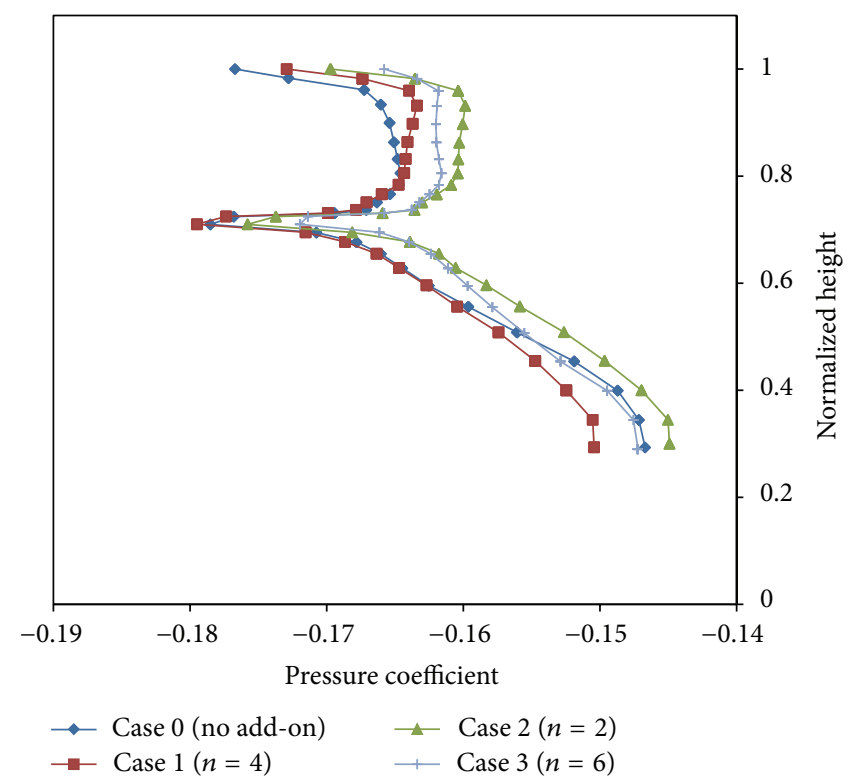

FIGURE 10: Pressure coefficient on the back surface of the cabin. (Origin of the axes is displayed in Figures 7 and 8. ) 

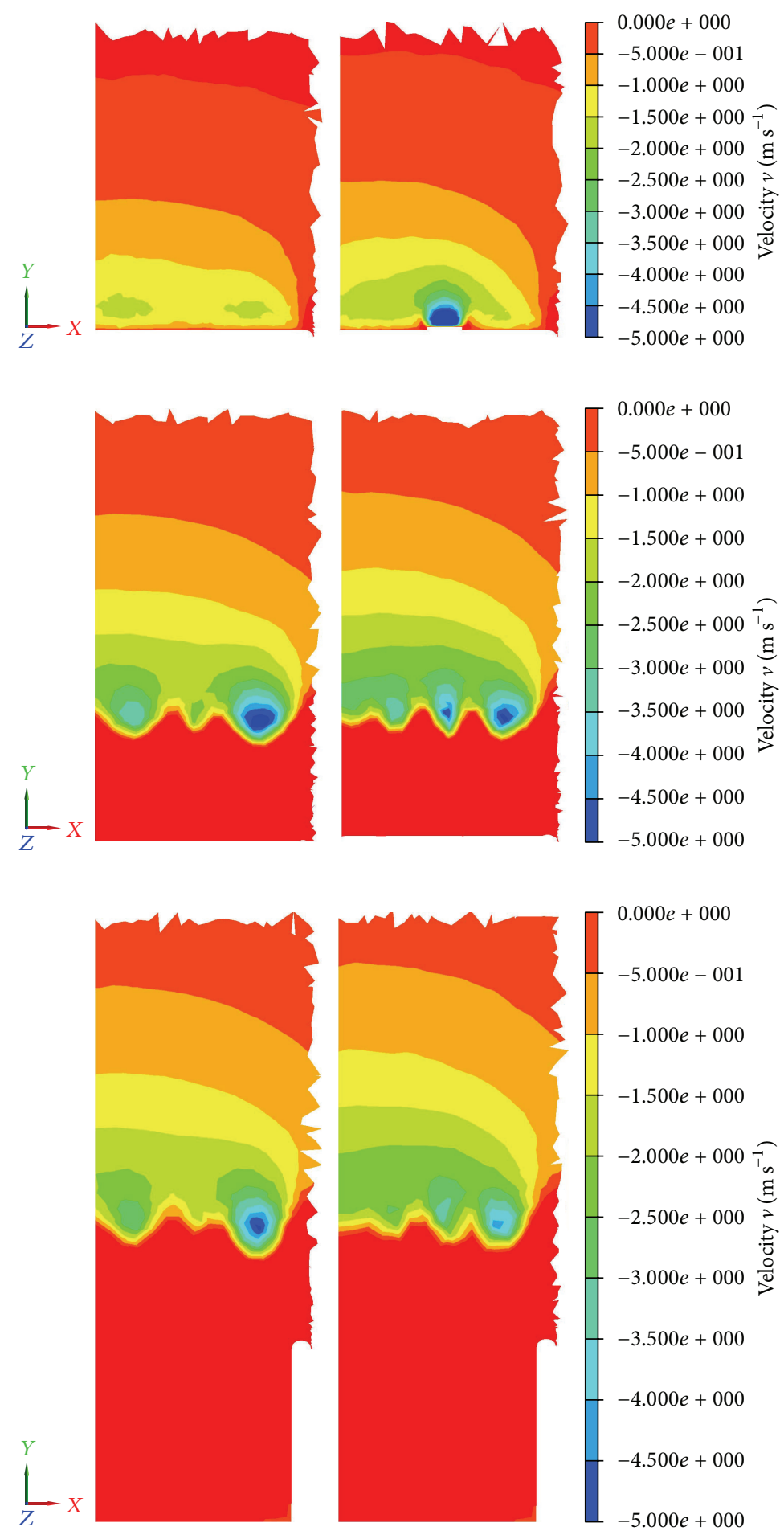

FIGURE 11: Velocity component over the planes of Figure 11, top (plane 1), middle (plane 2), and bottom (plane 3). Left column (plain configuration), right column (configuration with two bumps).

for the configuration with bumps). Similar pattern is also noticed in the second and third slide of the same figure. This deflection is also indicated in Figure 13 near the cabin back where we show the velocity vectors over the symmetry plane for both the plain configuration, Figure 13(a), and the configuration with two bumps, Figure 13(b); the velocity vector heads near the cabin back can be seen directing downward. In addition, we displayed the pressure distribution over the planes indicated in Figure 14; the contour plots of the pressure over these planes are shown in Figure 15. The core of the bed recirculating flow had shifted slightly away from the cabin back as indicated by the low pressure area (blue color) 


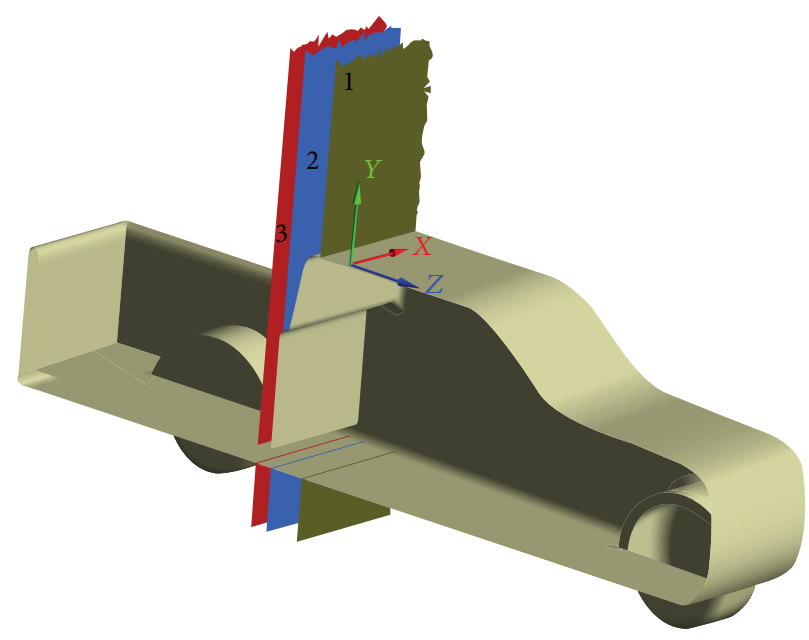

Figure 12: Planes of reference for Figure 11.

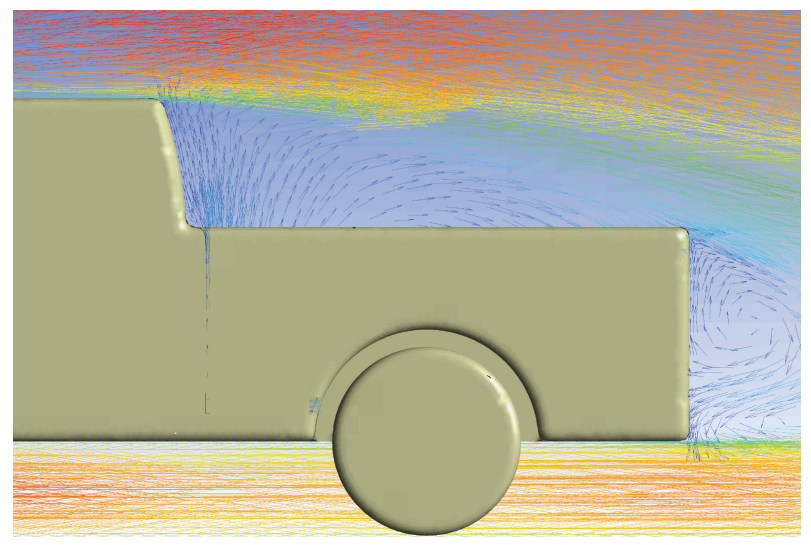

(a)

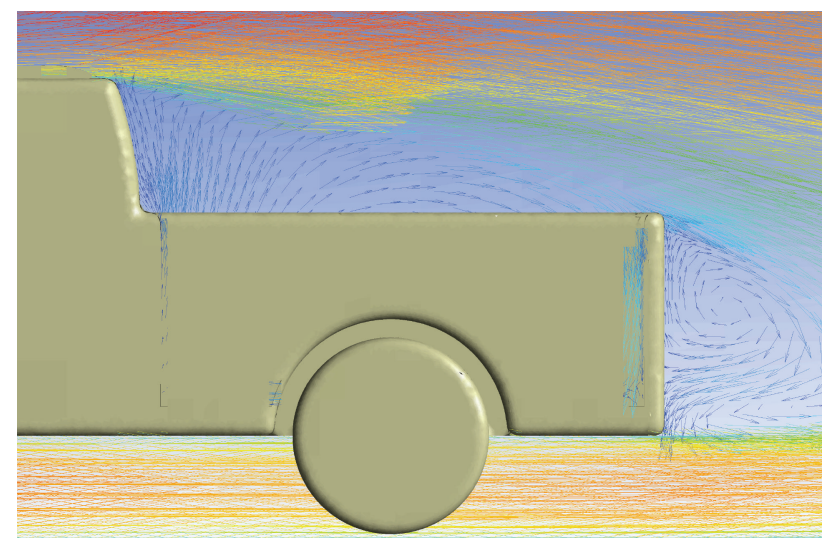

(b)

FIGURE 13: Velocity plot over the symmetry plane. (a) Without bumps, (b) with two bumps.

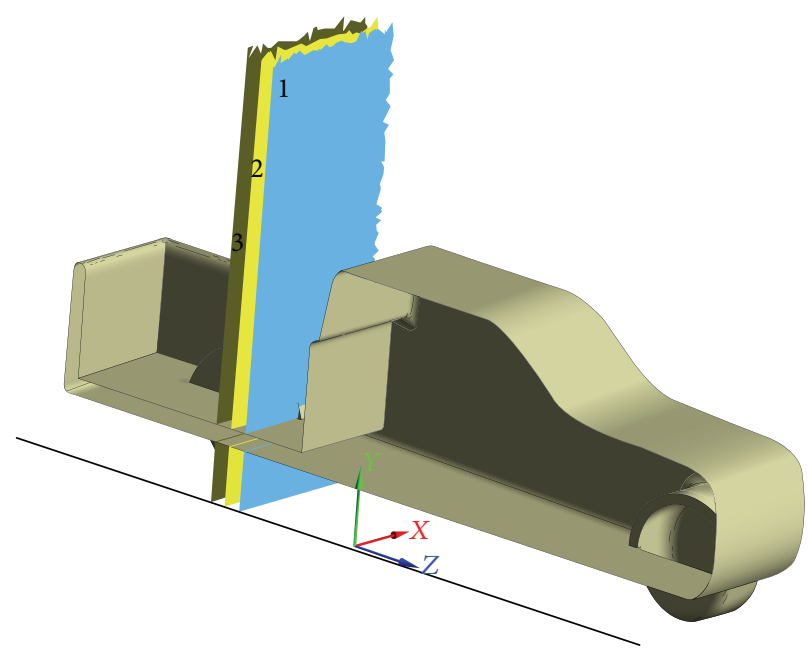

FIGURE 14: Planes of reference for Figure 15. 


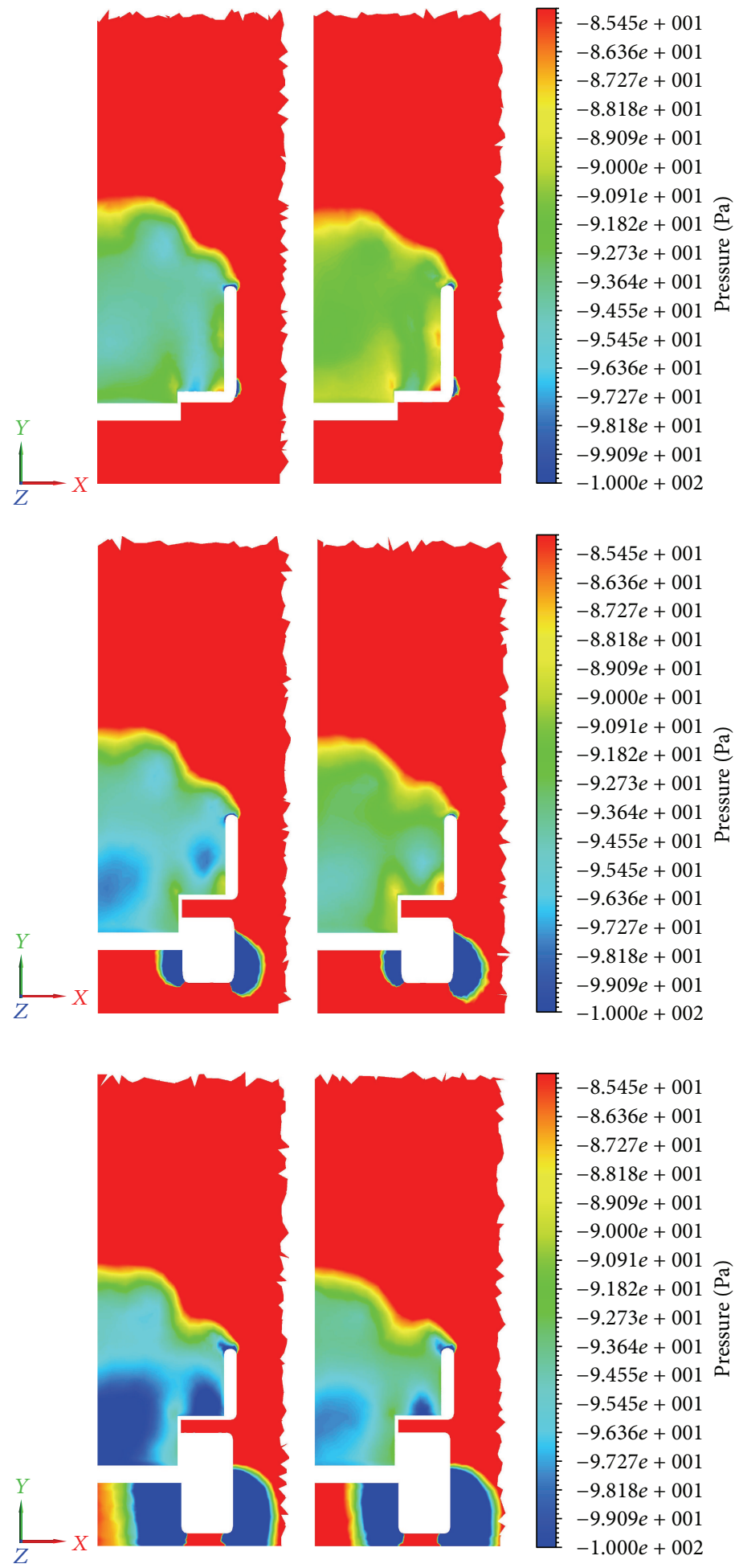

FIGURE 15: Pressure distribution over the planes of Figure 14, top (plane 1), middle (plane 2), and bottom (plane 3). Left column (plain configuration), right column (configuration with two bumps). 


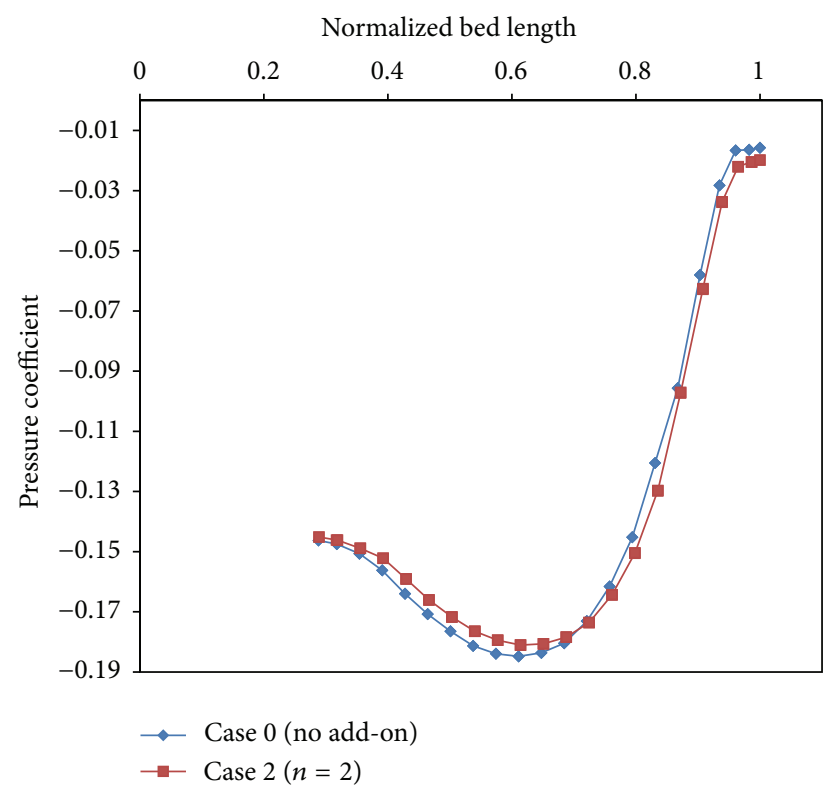

Figure 16: Pressure coefficient on the bed floor surface in the symmetry plane. (Origin of the axes is displayed in Figures 7 and 8. )

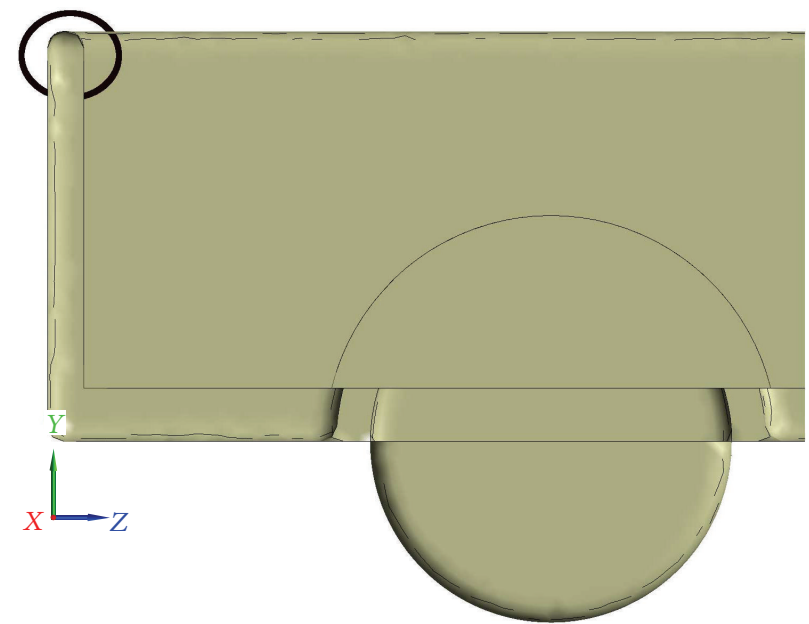

FIGURE 17: Close in on the area of interest in Figure 18.

corresponding to a farther location for the configuration with bumps than for the plain configuration. Overall, the flow deflection caused the tapering of the recirculating flow which led to the displacement of its core slightly farther from the cabin. This displacement was responsible for the pressure buildup behind the cabin and also explains the slight increase in the pressure coefficient on the bed surface in the proximity of the cabin compared to the plain geometry as indicated in Figure 16.

Because of the flow deflection caused by the bumps, the bed flow attachment over the tailgate had slightly moved toward the inside of the bed. This can be justified by comparing the turbulence intensities of the plain configuration and the configuration with two bumps over the upper part of the tailgate as indicated in Figure 17. The local coordinate frame shown in the figure has its origin on the road and its
$Z$-coordinate is measured from the outside surface of the tailgate. We summed the turbulence intensities on the upper part of the tailgate and over parallel grid lines with equal $Y$ values and then displayed the results in Figure 18. According to this chart, the turbulence intensity increases as we move toward the bed recirculating flow; however it becomes slightly more intense for the configuration with bumps near the inside surface of the tailgate. This indicated that the center of the dispersed flow over the tailgate had moved toward the bed recirculating flow for the configuration with two bumps. The displaced attachment enabled more downwash of the bed flow to fall into the wake, and thus the size of the reverse flow in the wake was reduced which accordingly improved the pressure behind the tailgate. This is also confirmed in Figure 19 where we summed the pressure over several parallel grids in the wake behind the tailgate as indicated in Figure 20. 


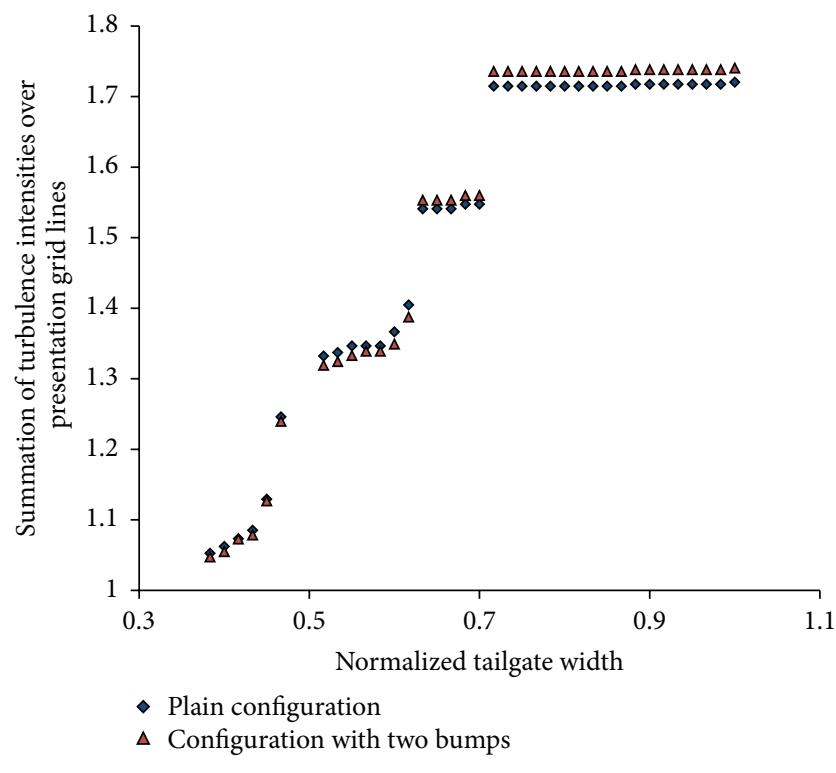

FIgURE 18: Accumulated turbulence intensity over the tailgate.

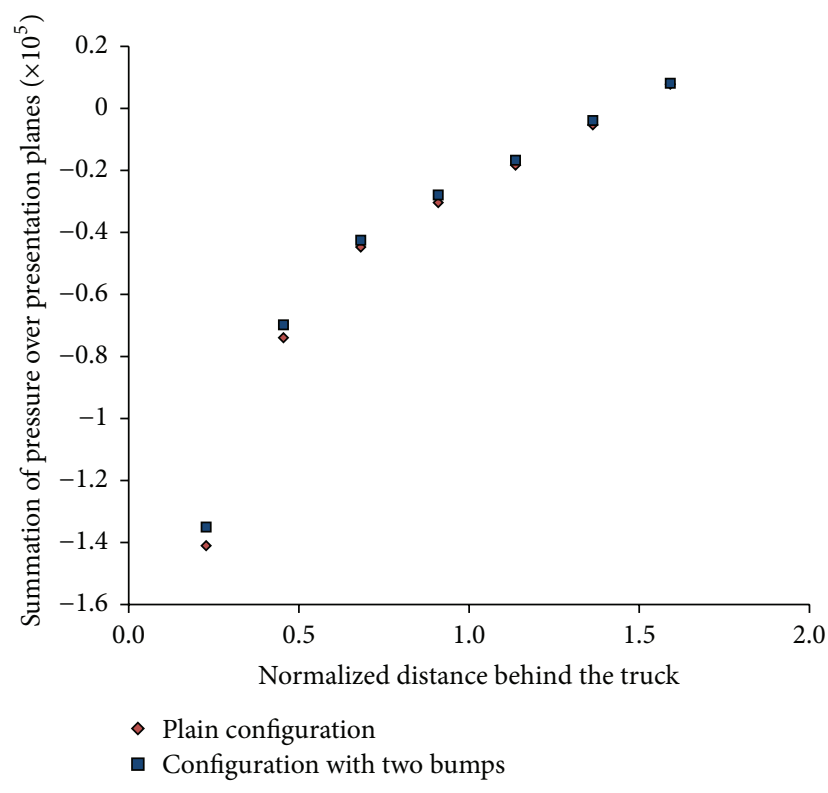

FIGURE 19: Summation of pressure over 7 presentation planes.

The overall pressure of the configuration with two bumps is slightly larger and asymptotically matches the pressure of the plain configuration far behind the wake.

\section{Conclusion}

We investigated and optimized the effect of multiple bumps placed in the rear end of the cabin roof on the overall aerodynamic drag reduction for a generic model of a commercial truck. The numerical method combined automatic redesign of the add-ons, simulation, and optimization using a globalized form of the Taguchi method. The numerical optimization showed that drag reduction can be achieved

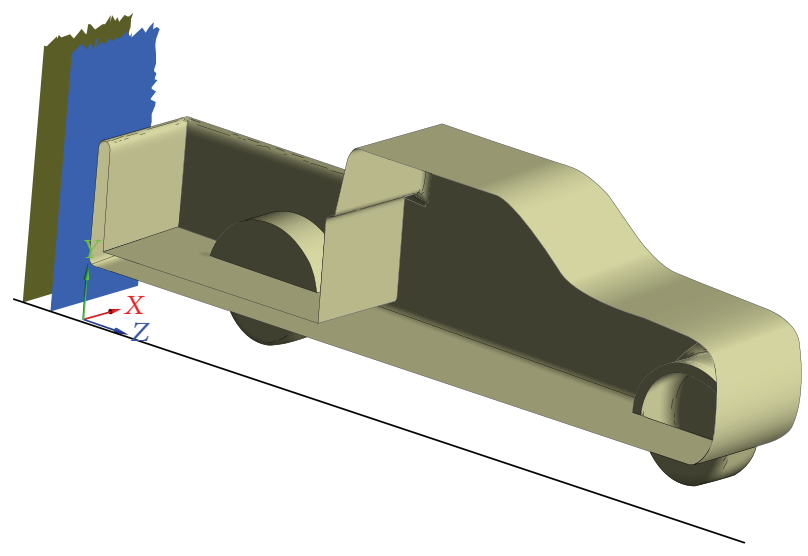

Figure 20: Planes of reference for Figure 19.

at different values of the design parameters with an overall expected reduction between 6 and $10 \%$. Overall, the bumps increased the cabin surface pressure coefficient and displaced the attachment of the bed flow over the tailgate toward the cabin, eventually reducing the size of the recirculating flow behind the tailgate and improving the pressure there.

Because several factors were involved in the design process and since their interactions were not known, an analysis of variance was omitted. A choice was made between the proposed optimum using Taguchi method and the best current vertex. The technique could be improved if one decided to analyze the interactions and devise a way to identify the optimum during the orthogonal array optimization.

\section{Appendix}

\section{Variable Variance Probability (VVP) Density}

The variable variance probability (VVP) density $[23,24]$ is based on the minimum distance to the points already sampled and is represented as

$$
\begin{aligned}
& \Phi(x)=\frac{1}{\sqrt{2 \pi} \sigma}\left(1-e^{d_{\min }^{2} / 2 \sigma^{2}}\right), \\
& d_{\min }=\underbrace{\min }_{i=1, \ldots, m}\left\{d_{i}=\sqrt{\sum_{k=1}^{n}\left(\frac{x_{k, i}-x_{k}}{x_{k, u}-x_{k, l}}\right)^{2}}\right\},
\end{aligned}
$$

where $\Phi(x)$ is the sampling probability of a point $x, n$ is the number of design variables, $x_{i}$ is a point previously sampled, and $m$ is the number of points already sampled. Length $d_{i}$ is the nondimensional distance between point $x$ and point $x_{i}$.

The variance of the normal probability density, which is updated in each restart, is given by

$$
\sigma=\frac{1}{3 \sqrt[n]{m}}
$$

The variance is gradually decreasing when the number of sampled points is increased. 


\section{Conflict of Interests}

The authors declare that there is no conflict of interests regarding the publication of this paper.

\section{Acknowledgment}

The first author would like to express his gratitude to the Office of Research and Grants at the University of Central Oklahoma (UCO) for the financial support during this research.

\section{References}

[1] W. H. Hucho, Aerodynamics of Road Vehicles, SAE International, 4th edition, 1998.

[2] G. Sovran, T. Morel, and W. T. Mason, Aerodynamic Drag Mechanisms of Bluff Bodies and Road Vehicles, Plenum Press, New York, NY, USA, 1978.

[3] W. H. Hucho and G. Sovran, "Aerodynamics of road vehicles," Annual Review of Fluid Mechanics, vol. 25, pp. 485-537, 1993.

[4] K. Cooper, "Pickup trucks aerodynamics-keep your tailgate up," SAE Paper 2004-01-1146, SAE International, 2004.

[5] J. Ha, S. Jeong, and S. Obayashi, "Flow characteristics of a pickup truck with regard to the bed geometry variation," Proceedings of the Institution of Mechanical Engineers Part D: Journal of Automobile Engineering, vol. 224, no. 7, pp. 881-891, 2010.

[6] J. Ha, S. Jeong, and S. Obayashi, "Drag reduction of a pickup truck by a rear downward flap," International Journal of Automotive Technology, vol. 12, no. 3, pp. 369-374, 2011.

[7] Z. Yang and B. Khalighi, "CFD simulation for flow over pickup trucks," SAE Paper 2005-01-0547, SAE International, 2005.

[8] A. Al-Garni, L. Bernal, and B. Khalighi, "Experimental investigation of the near wake of a pick-up truck," SAE Paper 2003-010651, SAE International, 2003.

[9] M. Gad-El-Hak, "Modern developments in flow control," Applied Mechanics Reviews, vol. 49, no. 7, pp. 365-379, 1996.

[10] M. Gad-El-Hak, A. Pollard, and J. Bonnet, Flow Control: Fundamentals and Practices, Springer, Berlin, Germany, 1998.

[11] S. S. Collis, R. D. Joslin, A. Seifert, and V. Theofilis, "Issues in active flow control: theory, control, simulation, and experiment," Progress in Aerospace Sciences, vol. 40, no. 4-5, pp. 237289, 2004.

[12] T. R. Bewley and S. Liu, "Optimal and robust control and estimation of linear paths to transition," Journal of Fluid Mechanics, vol. 365, pp. 305-349, 1998.

[13] P. Cathalifaud and P. Luchini, "Optimal control by blowing and suction at the wall of algebraically growing boundary layer disturbances," in Proceedings of the IUTAM Laminar-Turbulent Symposium, W. Saric and H. Fasel, Eds., pp. 307-312, Sedona, Ariz, USA, September 2002.

[14] G. Fournier, S. Bourgois, S. Pellerin, L. Ta Phuoc, J. Tensi, and R. El Jabi, "Wall suction influence on the flow around a cylinder in laminar wake configuration by large eddy simulation and experimental approaches," in 39e Colloque d'Aérodynamique Appliquee, Controle des Ecoulements, pp. 22-24, Paris, France, Mars 2004.

[15] J.-L. Aider, J.-F. Beaudoin, and J. E. Wesfreid, "Drag and lift reduction of a 3D bluff-body using active vortex generators," Experiments in Fluids, vol. 48, no. 5, pp. 771-789, 2010.
[16] M. Koike, T. Nagayoshi, and N. Hamamoto, "Research on aerodynamic drag reduction by vortex generators," Mitsubishi Motors/Technical Review, no. 6, pp. 1-16, 2004.

[17] R. K. Roy, Design of Experiments Using Taguchi Approach: 16 Steps to Product and Process Improvement, John Wiley \& Sons, New York, NY, USA, 2001.

[18] P. J. Ross, Taguchi Techniques for Quality Engineering, McGrawHill, New York, NY, USA, 1988.

[19] R. S. Rao, C. G. Kumar, R. S. Prakasham, and P. J. Hobbs, “The Taguchi methodology as a statistical tool for biotechnological applications: a critical appraisal," Biotechnology Journal, vol. 3, no. 4, pp. 510-523, 2008.

[20] W. Cui, X. Li, S. Zhou, and J. Weng, "Investigation on process parameters of electrospinning system through orthogonal experimental design," Journal of Applied Polymer Science, vol. 103, no. 5, pp. 3105-3112, 2007.

[21] S. Chen, X. Hong, and C. J. Harris, "Sparse kernel regression modeling using combined locally regularized orthogonal least squares and D-optimality experimental design," IEEE Transactions on Automatic Control, vol. 48, no. 6, pp. 1029-1036, 2003.

[22] W. Zhou, X. Zhang, M. Xie, Y. Chen, Y. Li, and G. Duan, "Infrared-assisted extraction of adenosine from radix isatidis using orthogonal experimental design and LC, Chromatographia, vol. 72, no. 7-8, pp. 719-724, 2010.

[23] M. A. Luersen and R. Le Riche, "Globalized nelder-mead method for engineering optimization," Computers and Structures, vol. 82, no. 23-26, pp. 2251-2260, 2004.

[24] H. Ghiasi, D. Pasini, and L. Lessard, "Constrained globalized Nelder-Mead method for simultaneous structural and manufacturing optimization of a composite bracket," Journal of Composite Materials, vol. 42, no. 7, pp. 717-736, 2008.

[25] M. Lanfrit, "3. Meshing," in Best Practice Guidelines for Handling Automotive External Aerodynamics with FLUENT, Version 1.2, Fluent Deutschland GmbH Birkenweg, Darmstadt, Germany, 2005. 

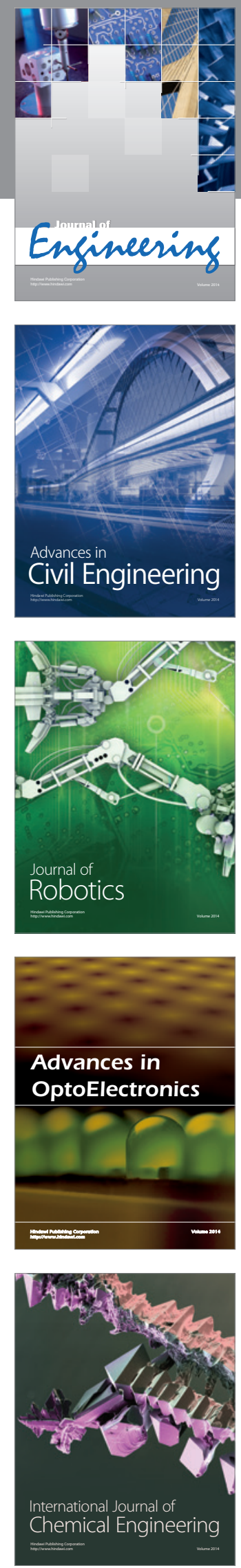

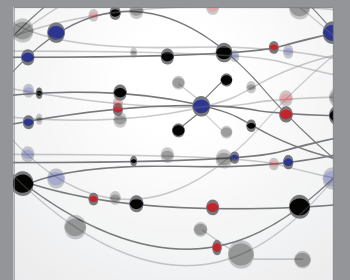

The Scientific World Journal
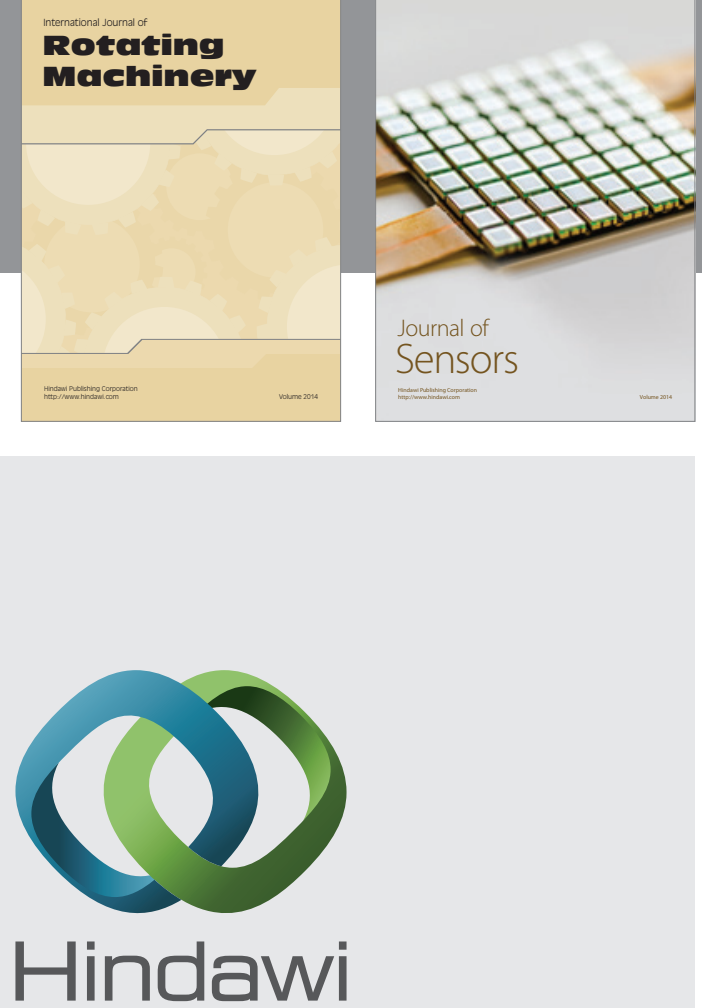

Submit your manuscripts at http://www.hindawi.com
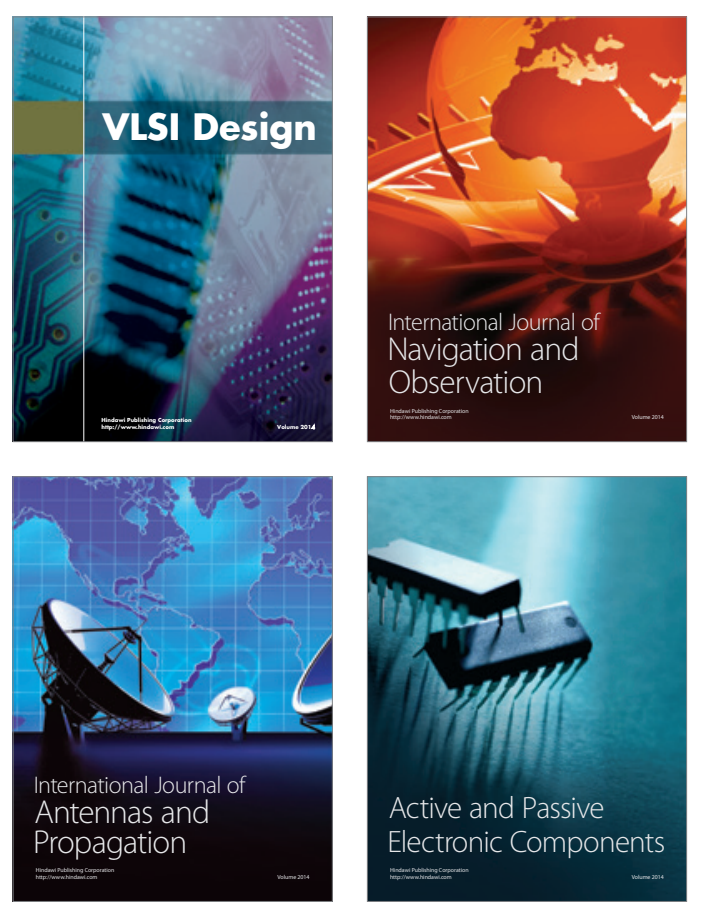
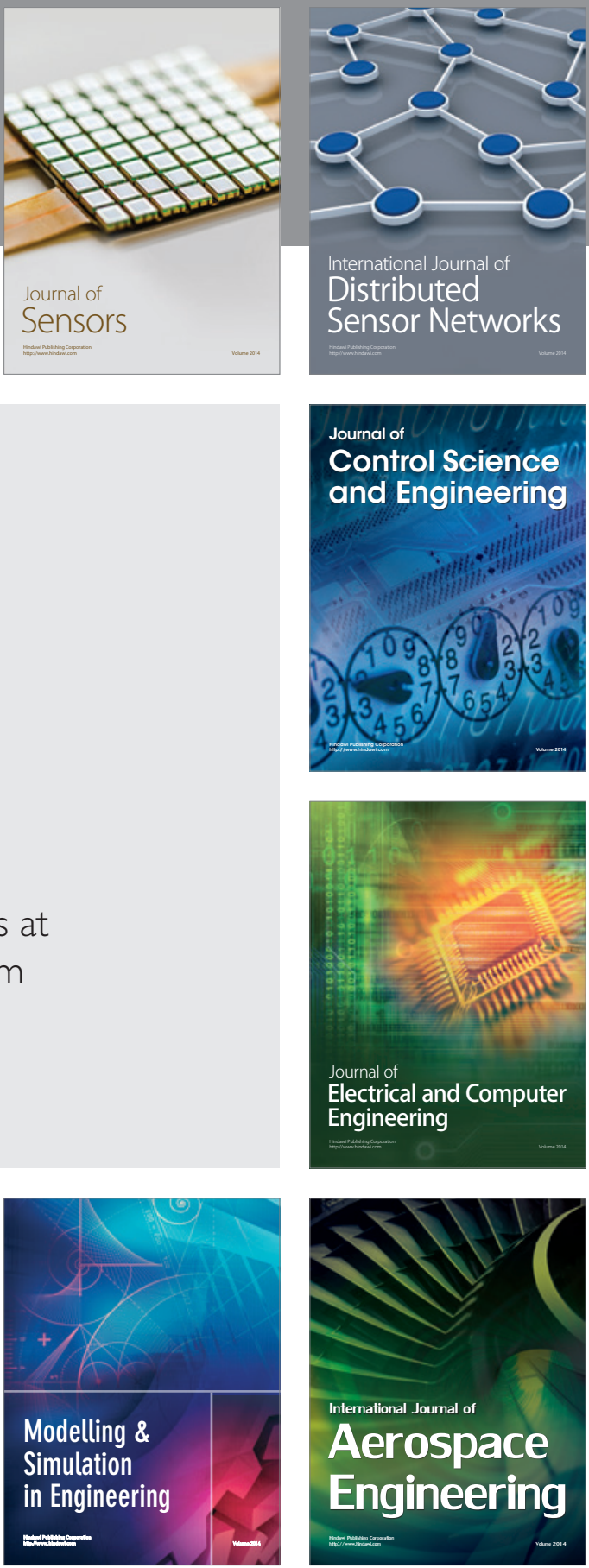

Journal of

Control Science

and Engineering
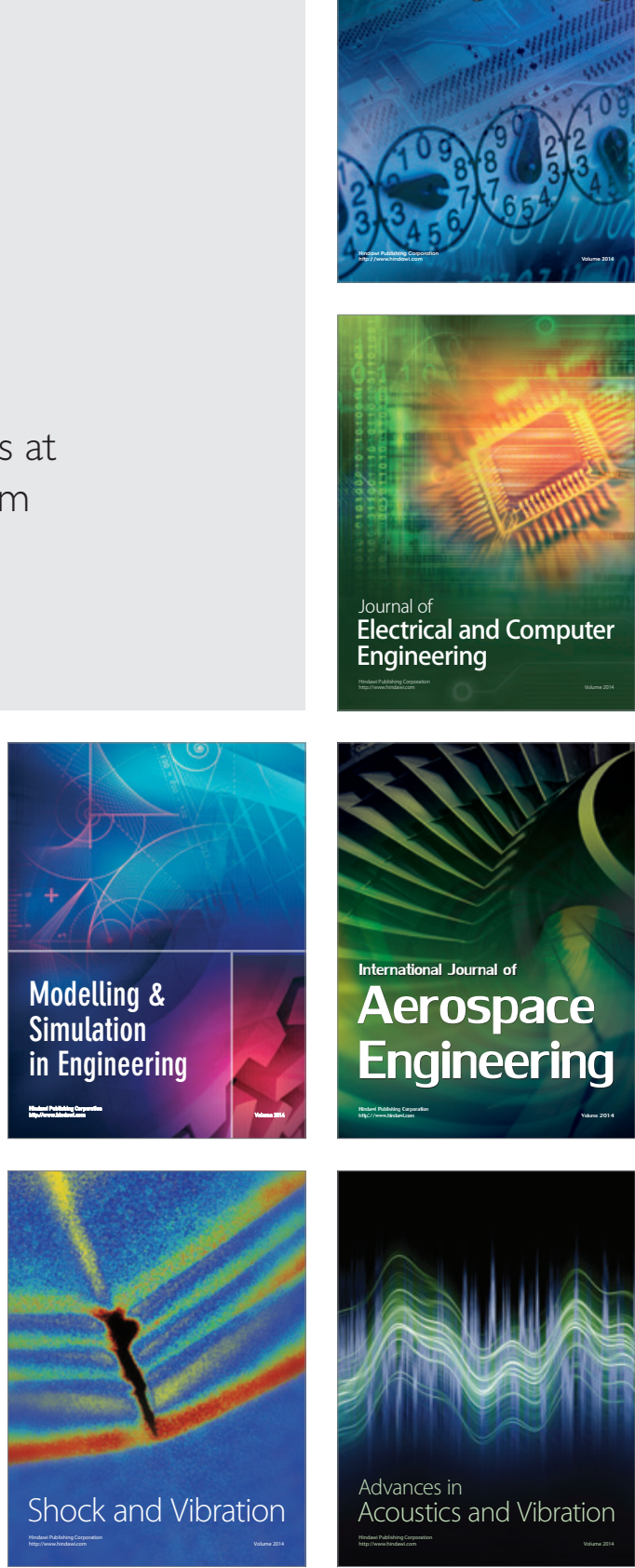